\title{
Asistencia técnica, la solución de la crisis de identidad de las cooperativas agrarias
}

\section{Agricultural extension, the solution of the identity crisis of cooperatives}

\author{
Amanda Vargas Prieto ${ }^{1}$
}

Recibido para publicación: Mayo 5 de 2017 - Aceptado para publicación: Noviembre 23 de 2017

\begin{abstract}
RESUMEN
La aparición de grupos cooperativos formados por cooperativas tradicionales y filiales industriales causó una crisis de identidad de este tipo de organizaciones a causa de un rompimiento del vínculo de los asociados con su cooperativa. En este artículo se analiza el papel de la asistencia técnica en el fortalecimiento de la relación entre la cooperativa y sus asociados. Un método cualitativo basado en el estudio de caso de cinco grupos cooperativos nos permitío estudiar las interacciones entre las comunidades cognitivas de las cooperativas a través de un nuevo modelo de gestión del conocimiento que se caracteriza por la interacción entre los grupos funcionales y las comunidades de práctica: el Modelo 3 que se realiza en el servicio de asistencia técnica a los productores agrarios. Este articulo, demuestra que los técnicso actúan como traductores entre las dos comunidades cognitivas y esto facilita la resolución de la crisis de identidad de las cooperativas agrarias.
\end{abstract}

Palabras clave: Asesoría, asistencia Técnica, Extensión Agraria, Cooperativas Agrarias, Comunidades Cognitivas, Gestión del conocimiento.

\begin{abstract}
The emergence of cooperative groups created by companies with a co-operative status, upstream production, and private companies resulted in the rupture of their relationship with their members. This paper addresses the role of agricultural consultation in strengthening the relationship between cooperatives and their members in order to resolve the inherent identity crisis of such groups. A qualitative method based on the case study of five groups allowed us to study the interactions between communities through a new mode of knowledge management characterised by the interaction between functional groups and communities of practice: the Model 3. We show that agricultural advisors act as translators between the two cognitive communities and this facilitates the resolution of the identity crisis of agricultural cooperatives.
\end{abstract}

Key words: Agricultural Extension, Extension System, Agricultural Cooperatives, Cognitive Communities, knowledge management.

\footnotetext{
1* Ph.D investigadora postdoctoral del Consejo Latinoamerica de Ciencias Sociales - CLACSO. Profesora Asociada de la Universidad de La Salle, Facultad de Ciencias Económicas y Sociales, Bogota, Colombia. Cra. 5 No. 59A-44 - Tel: (571) 3488000 ext 1586. amvargas@ unisalle.edu.co
} 
Vargas - Asistencia técnica y cooperativas agrarias

\section{INTRODUCCIÓN}

Se ha demostrado cómo la aparición de grupos de organizaciones cooperativas ha permitido a las cooperativas agrarias adaptarse a los cambios económicos y garantizar así su desarrollo. Pero esto no deja de tener consecuencias para la relación cooperativaasociado (Vargas Prieto, 2014). El cambio en la estructura de las cooperativas agrarias tiene un efecto de distanciamiento, de complejidad y de confusión para los asociados (Forestier y Mauget, 2001). Con la integración de la cooperativa a un grupo empresarial, el asociado tiene la impresión de ser un peón más en un juego económico cuyas reglas están más allá de su alcance (Nicolas,1993). Hoy en día, las cooperativas a nivel mundial se encuentran en un modelo difuso que combina los modos de gobernanza accionarial y asociativo, lo que es difícil de entender para los asociados. Se sienten alejados de los centros de decisión y les resulta difícil identificarse con estas nuevas estructuras (Duvaleix etal., 2003; Forestier y Mauget, 2001).

La crisis de identidad que afectó a los grupos cooperativos agrarios franceses (Vargas Prieto, 2013) plantea preguntas sobre el mantenimiento de las relaciones con los asociados y cómo se refleja en el funcionamiento de la cooperativa. Según Côté (2007; 2009), el reto para los grupos cooperativos agrarios es mostrar que pueden generar, con sus acciones económicas, resultados portadores de valor agregado para sus asociados con respecto a las empresas que no hacen parte del sector solidario.

En las cooperativas agrarias, se hace la puesta en común de recursos para facilitar o desarrollar la actividad económica y para mejorar o incrementar los resultados de su actividad. Por lo tanto, la cooperativa ofrece servicios y productos para facilitar la sostenibilidad de la actividad de sus miembros. Ellos son responsables de su cooperativa y se basan en principios éticos y de transparencia. Las cooperativas también están involucradas en el desarrollo territorial, son organizaciones democráticas controladas por sus miembros quienes participan activamente en el establecimiento de políticas y en la toma de decisiones. Los hombres y mujeres elegidos como representantes de los miembros son responsables ante ellos. La gestión de una cooperativa se basa así en el diálogo entre los empleados y asociados, para mantener el equilibrio entre la satisfacción de las necesidades e intereses de la cooperativa y de sus asociados. Al ser socios y clientes, la cooperativa debe encontrar soluciones que concilien la salud financiera de la empresa y la satisfacción de los asociados con el fin de mantener su negocio y participar en el desarrollo de las comunidades.

En un contexto de evolución del entorno reglamentario mundial; relacionado al acceso de los mercados y los subsidios, al cumplimiento de las normas medioambientales, de seguridad alimentaria, de salud, del bienestar animal y mantenimiento de los suelos, estos se convierten en criterios decisivos para la continuación de una actividad agraria productiva, pero es difícil de predecir y controlar (Barkaoui y Butault, 2004; Lefer, 1997; LeTheule y Litvan,1993). Para lograr este objetivo, la Comunidad Europea decidió establecer sistemas de extension agrícola a nivel nacional para responder a estos nuevos requisitos reglamentarios (véase el Reglamento CE N $\left.{ }^{\circ} 1782 / 2003\right)$. En Colombia en el marco del acuerdo para la terminación del conflicto y la construcción de una paz estable y duradera (2016), en el punto 1 sobre una "reforma rural integral" se reconoce la necesidad de que el gobierno ponga a disposición de los hombres y mujeres beneficiarios del Fondo de Tierras, entre otras cosas, la asistencia técnica en el marco de los Programas de Desarrollo Agrario Integral con Enfoque Territorial (p. 13). Luego en el punto 1.3.3 del acuerdo al identificar los estímulos necesarios para desarrollar la economía solidaria, se encuentra "el fortalecimeinto de las capacidades productivas y de las condiciones de acceso a los instrumentos 
de desarrollo rural (medios de producción, asistencia técnica, formación y capacitación, crédito y comercialización, entre otros)" (p. 24). La asistencia técnica a las explotaciones se convierte entonces en una de las formas clave de la adaptación de nuevos sistemas de cultivo a nivel mundial. Esta asistencia debe ser capaz de cumplir con los requisitos reglamentarios, manteniendo un alto nivel de producción y garantizando la renta de los agricultores (Remy et al., 2006).

Las cooperativas agrarias por su especificidad, están en mejores condiciones para desarrollar la actividad de la asistencia técnica. En efecto, se consideran como principales actores de la difusión de las nuevas prácticas o sistemas agrícolas en Francia, por ejemplo (Meynard, 1998). Por otra parte, es en el objetivo de acompañamiento, apoyo y desarrollo de la actividad de sus miembros que la cooperativa es creada. Sin embargo, a pesar de la legitimidad de las cooperativas de asumir la actividad de asistencia técnica, pocos estudios han examinado esta actividad (Faure et al., 2011). Teniendo en cuenta la crisis de identidad causada por los cambios estructurales de las cooperativas, y por otro lado, la creciente necesidad de los miembros de la cooperativa a responder a los cambios en la política agrícola mundial, este artículo se propone llenar este vacío. Por lo tanto, nos interesa comprender ¿cómo la asistencia técnica contribuye al fortalecimiento de la relación cooperativa-asociado para resolver la crisis de identidad de los grupos cooperativos agrarios?

\section{MATERIALES Y MÉTODOS}

En cuanto a la metodología, se optó por un enfoque cualitativo basado en estudio de casos de grupos cooperativos agrarios franceses para hacer frente a nuestra problematica. A falta de datos y estudios empíricos sobre asistencia técnica, esta elección metodológica nos permitió estudiar en profundidad y muy finamente la organización de la actividad de asisitencia técnica de diversos grupos cooperativos. De hecho, el enfoque exploratorio y metodología cualitativa fue elegido debido a la falta de información sobre este tema. La interacción con el objeto de estudio nos permitió comprender la realidad de la asistencia técnica en las cooperativas y su papel en la resolución de la crisis de identidad.

El estudio de caso se realizó sobre una muestra de 5 grupos cooperativos representativos en Francia. Respondiendo a criterios teóricos de representatividad, variedad, equilibrio y potencial de descubrimiento, asi estudiamos grupos polivalentes y especializados en diversos tipos de producción y ubicados geográficamente en todo el territorio francés. La movilización de múltiples fuentes de datos nos hizo posible identificar las características de la asistencia técnica de los grupos cooperativos y permitió la consideración de las estrategias más adecuadas para hacer frente a la crisis de identidad. A continuación presentamos la figura 1 que recapitula la metodología aplicada.

\section{RESULTADOS Y DISCUSION}

La metodología planteada nos permitió identificar en primer lugar, que la aparición de la asistencia técnica en las cooperativas corresponde con el período de emergencia de grupos cooperativos. El aumento del número de asesores, la diversificación de las actividades para prestar el servicio, la apertura de las tiendas de suministros y la mayor disponibilidad de los silos de almacenamiento en el periodo 2008-2011 concretamente justifican nuestra observación. También se observa que la actividad de asistencia técnica es muy especializada en sólo dos tipos de producción: ganadería y cereales. Esta estrategia puede ser guiada por la rentabilidad de este tipo de producción. En una gobernanza híbrida, el objetivo es maximizar el valor de los accionistas, incluyendo las filiales de producción. 


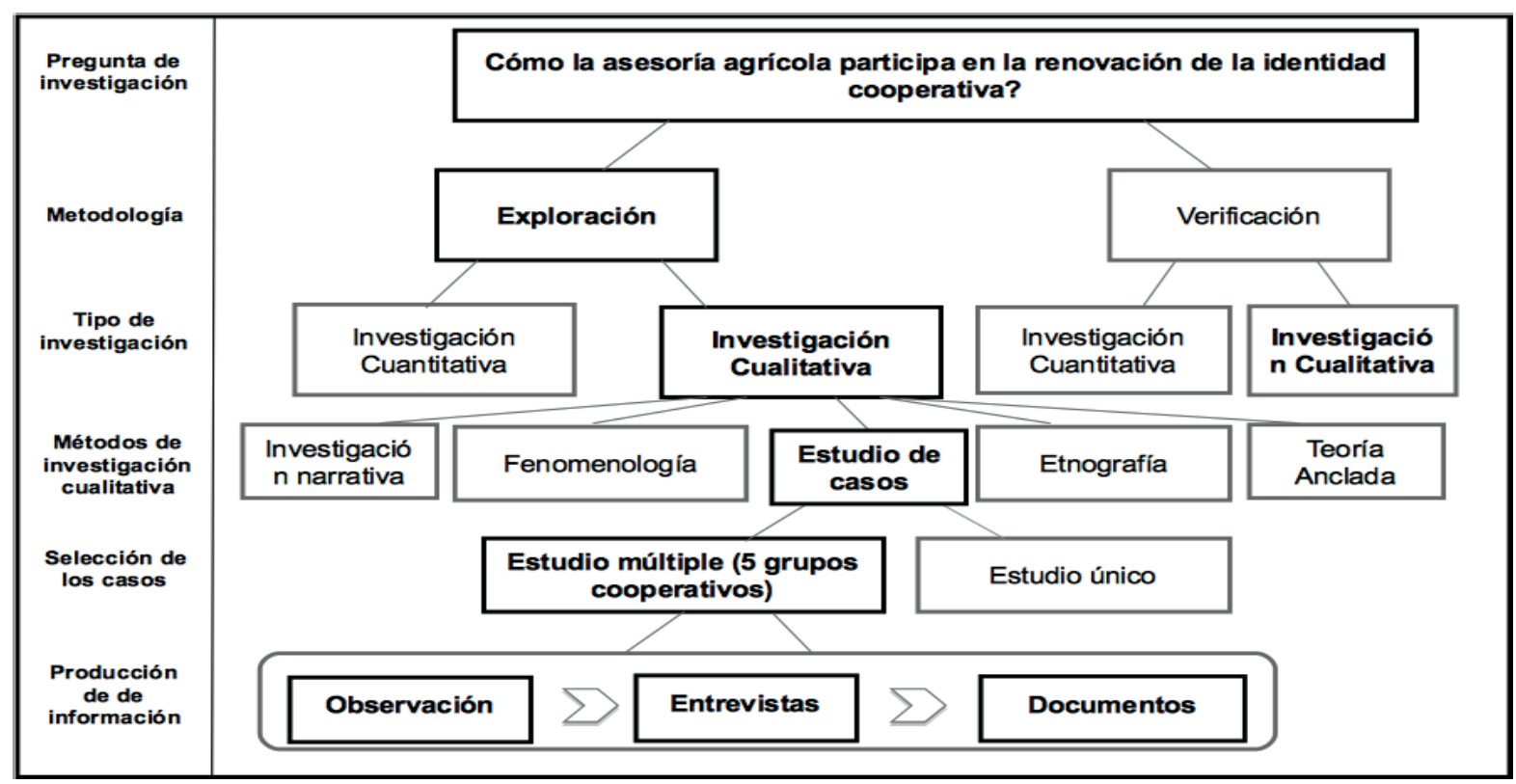

Fuente: Autor

Figura 1. Descripcion de la metodología utilizada en la investigación

A través de los estudios de caso, se identifican las características de la asistencia técnica en las cooperativas agrarias. Presentamos la evolución de la organización e identificamos los perfiles de los técnicos agrarios en los grupos cooperativos. Luego presentamos el impacto de las características de la asistencia técnica en el refuerzo del vínculo cocoperativaasociado. Por último, se presentan las estrategias de desarrollo de la asistencia técnica de los grupos cooperativos, para fortalecer el vínculo cooperativa - asociado.

La reconstrucción del territorio obligó a los grupos cooperativos a reorganizar la actividad de asistencia técnica. La estrategia de todas las cooperativas estudiadas fue fortalecer el apoyo técnico a los agricultores. Para entender cómo apareció esta actividad en las organizaciones, se construyeron los organigramas de cada grupo cooperativo partir de los datos disponibles. Una simplificación del organigrama general de los grupos cooperativos agrarios estudiados se muestra en la figura 2. La información estudiada nos ha permitido identificar que en los últimos dos años, se crearon dos departamentos encargados del back-office de la asistencia técnica en todos los grupos cooperativos.

Un departamento dedicado a la investigación

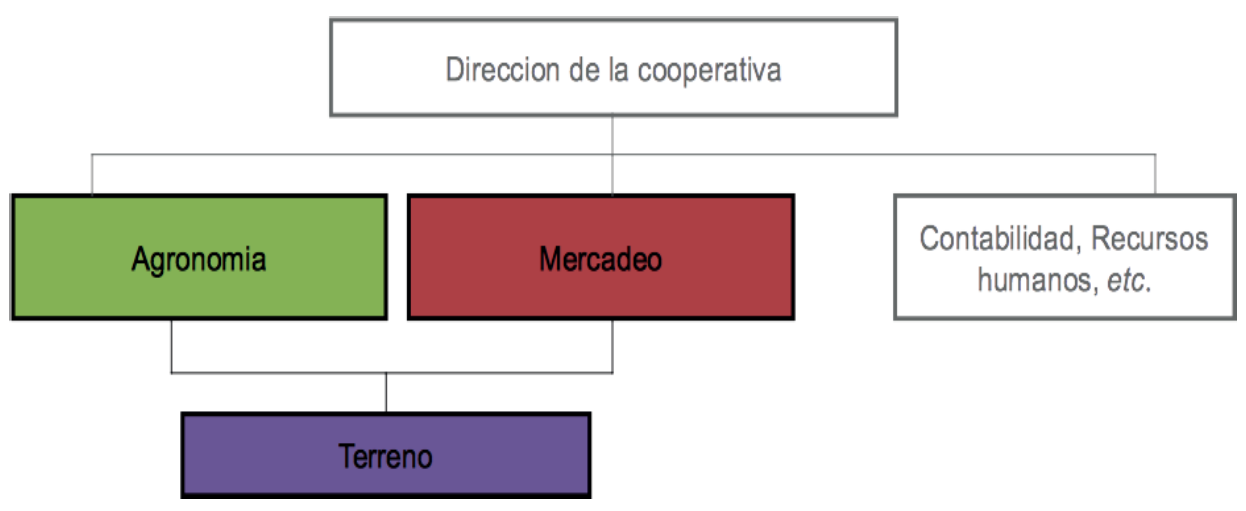

Fuente: Autor Figura 2. Organigrama standard de asistencia técnica de grupos cooperativos 
y experimentación encargada de mejorar el contenido de la asesoría, se Ilama Agronomía en la Figura 2 (verde) y un departamento encargado de crear la oferta de servicios, comunicación de los nuevos servicios y de la fijación de precios denominado marketing (rojo). Estos dos departamentos están directamente relacionados con los técnicos que realizan el front-office. El cuadro en color purpura en la figura 2 es denominado terreno y regrupa los técnicos que tienen relación directa con los asociados a las cooperativas. Los organigramas creados a través de los estudios de casos evidencian no sólo la labor de asesoria de los grupos cooperativos, sino también la diversidad de perfiles de los técnicos del departamento terreno (técnicos de terreno, investigación y experimentación, comercialización). Gracias al análisis de los datos identificamos cinco perfiles diferentes de asesores en la asistencia técnica cooperativa.

$\begin{array}{lcrr}\text { El } & \text { responsable de } & \text { portafolio } \\ \text { realiza } & \text { las siguientes } & \text { actividades: }\end{array}$

*Seguimiento de un grupo de agricultores con el fin de retener a maximizar el margen bruto de sus miembros, ofreciendo productos y servicios que satisfagan las necesidades de la producción. * Asesorar a los agricultores para el uso de los productos ofrecidos por las cooperativas (insumos y OAD )

* Llevar a cabo el seguimiento administrativo de los agricultores (la realización de contratos y órdenes de compra)

* Participar en la creación de la oferta comercial de la cooperativa

* Estar en contacto con losresponsables de los almacenes de suministro y con los jefes de silo. * Organizar reuniones técnicas y comerciales con los miembros de la cooperativa

* Promocionar la imagen de la cooperativa

* Respetar las normas de seguridad y calidad de la cooperativa

*Proponer y finalizar los contratos de producción animal
*Establecer las soluciones de financiación bajo el control de la cuenta de asociado

*Prospección entre los no miembros de la cooperativa

* Apoyar la creación de proyectos para la obtención de subvenciones y ayudas.

El asesor o técnico agro-ambiental realiza las siguientes actividades:

* Gestion administrativa de las OAD

* Llevar a cabo el desarrollo de las OAD

* Informar y formar a sus colegas sobre sus servicios

*Participar en la explotación y utilizacion de los datos técnicos y económicos recogidos en el marco de la trazabilidad de la produccion

*Mantener todas las estadísticas necesarias para cuantificar el impacto y la rentabilidad de las OAD

* Proporcionar un seguimiento administrativo del portafolio y revisar la normatividad

*Asesoramiento e información a los miembros

*Tratamiento de los subcontratistas potenciales

* Promocionar los servicios vendidos por la cooperativa

El asesor o técnico de investigación y experimentacion realiza las siguientes actividades:

*Busqueda de la información para diseñar programas experimentales

* Proponer protocolos de pruebas, participar en su aplicación y garantizar la síntesis de los resultados técnicos

*Desarrollar programas de difusión de resultados

* Desarrollar los itinerarios de los cultivos más rentables adaptadas a cada situación

* Desarrollar la opciones técnicas y económicas de los insumos

* Informar y animar la fuerza de ventas

* Realizar seguimiento técnico de la evolución de los cultivos a lo largo de la temporada

* Participar en las reuniones para presentar los 
Vargas - Asistencia técnica y cooperativas agrarias

expedientes técnicos

* Asegurar relaciones externas y de investigación para la firma de convenios con otras organizaciones

*Capacitarse para innovar y ser siempre precursor, seguir la reglamanetacione informar a los departamentos encargados

* Redactar los documentos y mensajes para los agricultores de acuerdo a su especialidad.

* Preparar las fichas técnicas necesarias para las campañas comerciales.

El responsable del almacen y de los silos realiza las siguientes actividades:

* Recibir a los miembros y/o clientes a la tienda: participa en las operaciones comerciales (Ilamadas, ventas en el almacen).

*Ejecutar el trabajo relativo a la operación del silo: la recepción, descarga, almacenamiento, clasificación, embalaje, envasado y transporte de toda la producción agrícola.

* Entregar todos los productos comprados

* Introducir la información al sistema

* Informar a su superior sobre la actividad de los almacenes o silos

* Garantizar la aplicación de las normas de seguridad y calidad

El responsable del almacen y de los silos realiza las siguientes actividades:

*Acompanar a los agricultores con asistencia preventiva

* Realizar intervenciones a problemas determinados

*Apoyo técnico-comercial a las fuerzas de venta de la cooperativa

* Redacción de documentos de información

*Apoyo a sus colegas sobre temas especializados

La identificación de las actividades de los distintos técnicos agrarios en los grupos cooperativos permite observar la proximidad que tienen con los asociados. Los técnicos que participan en el back-office se encuentran un poco más alejados que los que participan en el front-office. De hecho, los técnicos que participan en el back-office son los técnicos de investigación y experimentación (perfil 3) y técnicos agro-ambientales (perfil 2). Estos dos tipos de asesores son responsables de crear el contenido del back-office y la interacción con los asociados es poco frecuente o inexistente. Por otro lado, los técnicos que forman parte del front-office son los responsables de portafolio (perfil 1), los veterinarios (perfil 5), los responsables de los almacenes y de los silos (perfil 4). Su función es la de servir a los miembros, son los técncos del territorio que se encuentran de cara a cara con los asociados. Los técnicos del back-office informan y capacitan a los técnicos del front-office para homogeneizar el conocimiento y luego difundir la información a los asociados. Esta difusión de información se logra principalmente a través de las visitas individuales que pueden ayudar a fortalecer el vínculo con los asociados. Sin embargo las reuniones temáticas y el uso de las TIC también son importantes. Los grupos envían boletines informativos periódicos, correos electrónicos, etc. Las recomendaciones técnicas también están publicadas en la intranet de la cooperativa cuyos miembros tienen acceso. Sin embargo, podemos deducir que esta no participa en la construcción del vínculo cooperativaasociado, ya que no facilita los intercambios. Para comprender mejor el desarrollo de las operaciones de back-office $y$ front office, ubicamos los diferentes tipos de técnicos en el organigrama de un grupo cooperativo (Figura 3). Las características de la actividad de asistencia técnica dentro de los grupos cooperativos son las siguientes. En primer lugar, la participación de los asociados en la orientación de la asistencia técnica. En segundo lugar la diversidad de los servicios que ofrecen las cooperativas y por ultimo, el acceso igualitario al servicio técnico y la segmentación de la prestación del servicio.

Durante la visita a las cooperativas, se encontró que se llevan a cabo evaluaciones 


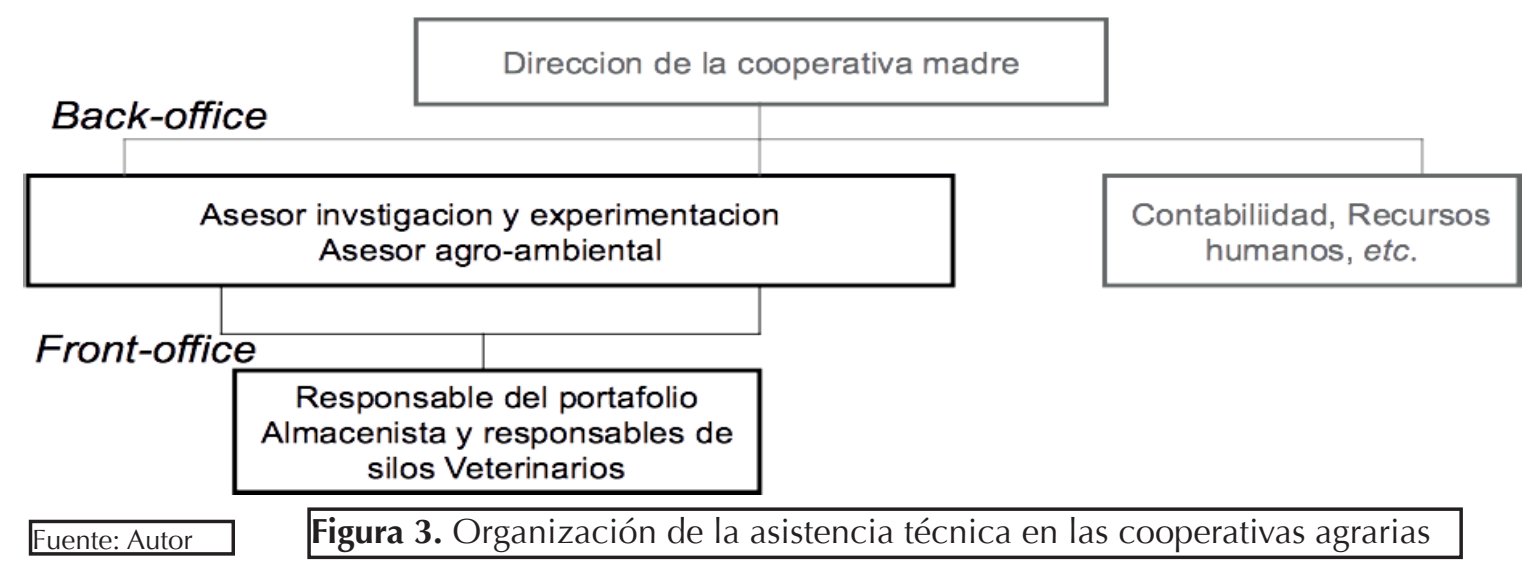

del servicio de asistencia técnica en la mayoría de las cooperativas. Así que decidimos investigar a fondo estos resultados. Todos los grupos cooperativos realizan encuestas una vez al año, en promedio, para identificar las preocupaciones de los miembros y sus expectativas de servicio. Dos estrategias de evaluación surgen de nuestros estudios de caso. Por un lado, los grupos especializados están desarrollando cuestionarios de las encuestas y los ponen a disposición en la intranet, lo que puede ser negativo para la construcción de vínculo cooperativa-asociado. De hecho, las encuestas de internet son voluntarias y, por tanto, limitados en número y sólo los participantes que utilizan esta herramienta pueden responder.

Una de las características que hemos observado durante las visitas de campo es la variedad de servicios más allá de la asistencia técnica. De hecho, los miembros están demandando cada vez más el desarrollo de OAD y el apoyo económico y estratégico de las operaciones. Para entender esta diversidad de servicios, se llevó a cabo la estandarización de la información recogida de los grupos cooperativos para desarrollar una lista de identificación de todos los grupos de servicios en nuestra muestra. Hicimos una lista de 76 servicios ofrecidos por los grupos cooperativos. Clasificamos estos servicios en tres tipos de acompañamiento. Así se agruparon en el acompañamiento técnico y productivo un total de 44 servicios relacionados con la gestión de enfermedades, parásitos y los incidentes de cultivos, manejo de fertilizantes, formación, beneficios en la reproducción y variedad. El segundo tipo de acompañamiento recoge 16 servicios relacionados con el apoyo al medio ambiente. Estos servicios están relacionados con el uso de pesticidas, los reglamentos de cría, seguimiento de la PAC, entre otros. El tercero es el acompañamiento económico y estratégico. Cuenta con 16 servicios relacionados con la formación económica y financiera para la gestión de la producción, conocimientos de informática y el seguimiento del software de gestión. Por lo tanto, los grupos cooperativos no solo acompañan a los asociados en la producción agricola. Se dan consejos sobre la asignación de recursos financieros y la gestión de las operaciones y asesoran sobre las nuevas regulaciones, para ayudar a los agricultores a hacer frente a los retos medioambientales. Una vez clasificados los servicios en los tres tipos de acompañamiento, analizamos el tipo de

Tabla 1. Disponibilidad de los servicios prestados en la asistencia técnica en las cooeprativas agrarias.

\begin{tabular}{lccccc}
\hline \multicolumn{1}{c}{ Tipos de acompañamiento } & \multicolumn{2}{c}{ Grupos polivalentes } & \multicolumn{3}{c}{ Grupos especializados } \\
\hline Grupos cooperativos & GC1 & GC5 & GC4 & GC3 & GC2 \\
Acompañamiento1 - Técnico y productivo & 29 & 36 & 33 & 27 & 26 \\
Acompañamiento 2 - Ambiental y reglamentación & 7 & 12 & 12 & 11 & 5 \\
Acompañamiento 3 - Economico y estrategico & 4 & 7 & 9 & 9 & 7 \\
Total servicios de asistencia técnica & 40 & 55 & 54 & 47 & 38 \\
\hline Fuente: Autor & & & &
\end{tabular}


Vargas - Asistencia técnica y cooperativas agrarias

apoyo que favorece cada grupo cooperativo. Esto es para mostrar la disponibilidad de estos servicios y asi identificar su estrategia. Esta información se presenta en la tabla 1.

De acuerdo con la tabla anterior, los grupos GC4 y GC5 son los grupos que tienen la mayor cantidad de servicios de asistencia técnica en su oferta en 2010. Los grupos GC1, GC4 y GC5 tienen una gran oferta de servicios en el acompañamiento 1 y 2 con respecto a los grupos GC2 y GC3. Los grupos especializados tienen más servicios en el tipo de acompañamiento 3 que los grupos polivalentes. Sin embargo, GC4 persigue el nivel de los grupos especializados con el acompañamiento 3. Por lo tanto, GC4 parece haber adoptado la mejor estrategia en el desarrollo de servicios de asistencia técnica.

Hay tres posibilidades de acceso a los servicios de asistencia técnica de los grupos cooperativos agrarios: un compromiso fuerte $y / o$ incluido en un paquete de servicio/producto, bajo nivel de compromiso y/o tarifas de los servicios separados de los productos, y un precio independiente al compromiso de asociado. De acuerdo con estas estrategias de precios, el intercambio con los asociados es más o menos fácil. Por ejemplo, si el acceso a los servicios se relaciona con el compromiso, los asociados no comprometidos tienen limitaciones para acceder al servicio de la asistencia técnica.

Los estudios de caso permiten identificar diferentes estrategias de desarrollo de esta actividad dentro de los grupos cooperativos. Estas estrategias específicas de los grupos cooperativos participan en la fidelidad de los miembros y mantiene el vínculo entre ellos y la cooperativa. Hemos identificado tres estrategias. La primera estrategia es el desarrollo de la experimentación y las asociaciones que responden a una necesidad de intercambio de conocimientos. La segunda estrategia se relaciona con el compromiso con las certificaciones de la asistencia técnica, garantías de la calidad del servicio. Una tercera estrategia es el desarrollo de las herramientas de difusión de la asesoria que buscan mejorar la comunicación.

A través de la movilización del marco teórico de la economía del conocimiento propuesto en el marco teórico. Se trata de hacer una lectura de la asistencia técnica y así mostrar que a través de las comunidades cognitivas, podemos entender cómo los grupos cooperativos pueden utilizar esta actividad para fortalecer el vìnculo con sus asociados.

En todos los grupos estudiados, son los técnicos de portafolio los que realizan la asistencia técnica. Por ejemplo, en GC2 hay 8 visitas por año para cada asociado, en el GC2 6 visitas, seguidos de GC4 con 5 visitas por año y GC5 con 4 visitas por año. El grupo, que tiene menos visitas anuales es GC3 con 3 visitas al año. Se ha encontrado que la creación de sitios de Internet y el uso de la intranet sirven para informar a los miembros. Por lo tanto, todos los grupos desarrollaron un módulo agronómico que está disponible en la extranet. Este módulo contiene toda la información técnica sobre los cultivos, y en algunos grupos, también hay AOD a disposición de los miembros. El uso de las TIC juega un papel importante en la difusión de la información en las cooperativas agrarias. Por último, boletines informativos se envían a todos los miembros con los resultados de los ensayos de variedades de cultivo o la introducción de nuevas técnicas.

El envío de estos boletines facilita la comunicación entre la cooperativa y sus miembros. La información contenida en este boletín no se limita a las cuestiones técnicas, también incluye información general sobre la cooperativa, lo que ayuda en la difusión de información estratégica del grupo cooperativo. Con respecto a la formación, encontramos la formación técnica para la aplicación de productos fitosanitarios, también sobre 
almacenamiento y conservación de cereales, las certificaciones, algunos elementos de la gestión y la relaizaciòn de los dossiers para acceder a las ayudas de la PAC, la gestión de riesgos, variedad de precios, análisis de mercado, el descubrimiento de Internet y extranet y, finalmente, el trabajo en equipo. Estas formaciones están involucrados en el desarrollo de habilidades como el liderazgo y el dominio de las herramientas informáticas. Estos cursos

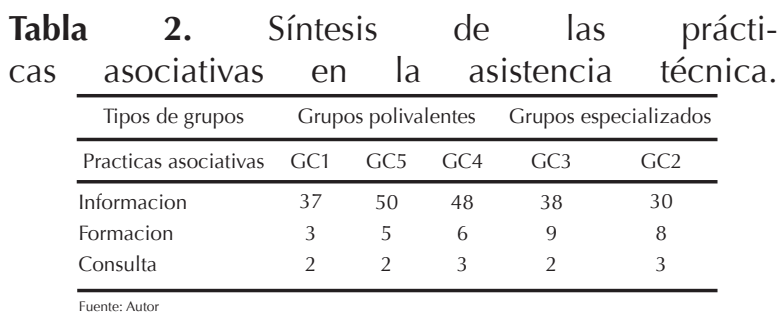

también se llevan a cabo por los diferentes tipos de técnicos dependiendo su especialidad. En la lista de servicios ofrecidos en la asistencia técnica no hay prácticas a priori de consulta de toma de decisiones. Sin embargo, se tienen en cuenta las asambleas generales que realizan las cooperativas para la toma de decisiones. Así que añadimos a la lista de prácticas asociativas la participación en los diferentes órganos de participación de los asociados en las decisiones de su cooperativa.

A continuación presentamos una tabla donde recapitulamos los resultados encontrados en cada grupo (Tabla 2). De acuerdo con la tabla anterior, la práctica asociativa más desarrollada es la información, seguido por la formación y la consulta. De hecho, los grupos cooperativos tienen muchos más servicios relacionados con la difusión de la información. Por último, incluso si la consulta ha estado siempre presente en los órganos colegiados a través de las asambleas, creemos que la aparición de grupos cooperativos también impulsó otras herramientas de consulta, tales como la evaluación de la asistencia técnica para adaptarse mejor al nuevo contexto agrícola. De hecho, pedir a los miembros su opinión sobre la efectividad del servicio de asistencia técnica puede adaptar su oferta a la demanda.

Como parte de las tres prácticas asociativas resaltadas en la asesoría técnica, existen interacciones entre los técnicos y los asociados en los tres casos (información, formación y consulta), hay intercambios entre los técnicos y asociados. Analizar estas interacciones a través del prisma de las comunidades cognitivas ayuda a comprender el impacto de la labor de la asistencia técnica sobre la relación cooperativa- asociado en los grupos cooperativos. Mediante el estudio identificamos grupos funcionales, los cuales surgieron durante el desarrollo de la actividad de asesoría técnica dentro de los grupos cooperativos.

Los grupos funcionales son la base de los modelos tradicionales de las organizaciones como, por ejemplo, marketing, finanzas, recursos humanos o contabilidad. Los grupos funcionales son las comunidades que tienen como objetivo la difusión del conocimiento (Cohendet et al., 2003). Los miembros son reclutados por la jerarquía de la empresa basada en el conocimiento homogéneo reconocido por un título. Por lo tanto, los miembros de los grupos funcionales tienen conocimiento homogéneo de un tema en particular. Enocntramos dos grupos funcionales alrededor de la actividad de asistencia técnica que se encargan de la experimentación y la comercialización. De hecho, estos dos grupos funcionales están formados por ingenieros, técnicos, profesionales con conocimientos en agricultura.

propósito de estos dos grupos funcionales es crear nuevas soluciones técnico-económicas que satisfagan las demandas de los miembros como se ha explicado anteriormente en los hechos estilizados. El primer grupo funcional es la agronomía. Este grupo es responsable de crear el contenido de la asistencia técnica y de difundir la información a los otros miembros de la organización. Esta comunidad se compone 
Vargas - Asistencia técnica y cooperativas agrarias

de asesores en el back-office (técnicos de investigación y experimentación y técnico agroambiental). Estos técnicos intercambian con los técnicos del front-office a través de la formación y la difusión de información a través de documentos tales como manuales técnicos destinados para ellos. Los intercambios que tienen ellos pueden producir nuevos conocimientos sin querer. Por lo tanto, este tipo de comunidad no sucede o no crea nuevos conocimientos en forma voluntaria, sino que garantiza su difusión entre los miembros de la comunidad. El segundo grupo funcional es la comercialización, formado por miembros con conocimientos en el mismo campo (comercialización). Su objetivo es la creación de una oferta de asesoria y una fijación de precios más atractivos para los usuarios. Esta comunidad es también responsable de la realización de encuestas a los asociados con el objetivo de identificar sus demandas y su opinión sobre la asistencia técnica. Una comunidad de práctica es toda comunidad que comparte conocimientos en un área específica, el objetivo es desarrollar habilidades o encontrar "mejores prácticas" o la solución a un problema (Brown y Duguid,1992; Lave y Wenger, 1991; Wenger,1998). Tres características de las comunidades de práctica son: el dominio de un campo de conocimiento, el hecho de que este conocimiento permita la interacción entre los miembros de la comunidad y que esta interacción conduce a la generación de recursos.

Las comunidades de práctica son para los miembros (Brown y Duguid,1992; Lave y Wenger ,1991). Mejoran las habilidades individuales a través del intercambio y la difusión de un directorio de recursos comunes (Wenger, 1998). A través de nuestros estudios de caso, hemos identificado un tipo de comunidad de práctica en los 5 grupos cooperativos, esta surge a través de la interacción entre los técnicos y los miembros.
De hecho, los técnicos y los asociados comparten la misma área de conocimiento: la producción agrícola. Los miembros de esta comunidad buscan nuevas soluciones para el mejoramiento de la producción. Las formas de interacción son las visitas individuales, sesiones de grupo, llamadas telefonicas, la distribución de documentos, el correo, SMS, etc. Esta interacción también conduce a la creación de nuevos recursos, incluidos los experimentos, las herramientas, las posibles soluciones a los problemas comunes, etc. La organización de la actividad de asistencia técnica agrícola dentro de los grupos cooperativos puede ser representada por grupos funcionales y comunidades de práctica. En la siguiente sección, nos centramos en las interacciones entre estos dos tipos de diferentes tipos de comunidades para la comprensión del modelo de gestión del conocimiento específico a los grupos cooperativos.

Deducimos de nuestros estudios de caso que el papel de los técnicos es el de la traducción entre las dos comunidades y asi promover la difusión y creación de conocimiento dentro de los grupos cooperativos agrícolas. A continuación, mostramos que es principalmente el técnico de portafolio el que participa en la intermediación de los diferentes tipos de comunidades dentro de los grupos cooperativos agrícolas.

El intercambio entre las dos comunidades se realiza a través de las prácticas asociativas. Las prácticas de información y de formación se realizan en el marco de la asistencia técnica y facilitan la difusión del conocimiento de los grupos funcionales a los agricultores $y$, por tanto a las comunidades de práctica (topdown), gracias a la intermediación de los técnicos. De hecho, los grupos funcionales envian documentos, informan y capacitan a los agricultores a través de la intervención de los técnicos o mediante el uso de las TIC.

Estas prácticas de información y formación 
ilustran el hecho de que las comunidades están unidas a través de la difusión de la información, como en cualquier otra organización. En el caso particular de los grupos cooperativos, los intercambios no sólo se basan en la difusión del conocimiento de una comunidad a otra, sino que también pueden ser la base para la creación de nuevos conocimientos a través de la interacción. La participación de los miembros de la asistencia técnica de los grupos funcionales (bottom-up) a través de la práctica de asistencia técnica (evaluación de la asistencia técnica, por ejemplo) permite un retorno de las comunidades de practica hacia los grupos funcionales de la cooperativa. En nuestro estudio de casos, hemos visto que los grupos funcionales de los grupos cooperativos tienen la capacidad de crear servicios de asistencia técnica a partir de los resultados de las evaluaciones hechas por los asociados; en un proceso de top-down, se extienden los conocimientos hacia los asociados.

Esta distribución se realiza principalmente por el técnico de portafolio que tiene contacto directo con los agricultores. Las prácticas asociativas de información, formación y consulta pueden entonces facilitar no solamente la difusión, sino también la creación de conocimiento dentro de los grupos cooperativos agrarios. Es a través de la consulta que los grupos cooperativos agrarios tienen canales de comunicación para concoer las necesidades de los miembros. La consulta se lleva a cabo por la retroalimentación de los asociados de manera virtual y también por la retroalimentación proporcionada a los técnicos. De hecho, como parte de la relación de servicio, los técnicos y los asociados intercambian conocimiento de diferentes tipos (tácito o codificado). Estos intercambios permiten a los técnicos recoger nuevos conocimientos que se devuelven a los grupos funcionales en un proceso de bottom-up.

La interacción entre los técnicos encargados del front-office y los técnicos del back-office capitaliza la informacion es decir resume, clasifica, indexa, en otras palabras, codifica el conocimiento. Estos conocimientos codificados son luego transferidos normalmente en forma de documentación técnica, a través del uso de las TIC y las herramientas de acompañamiento en la toma de decisiones (OAD). El análisis empírico del proceso de creación de conocimiento dentro de los grupos cooperativos agrarios, nos lleva a validar el modelo de creación de conocimiento 3 .

A continuación presentamos una figura 4 que hace explícito la creación de conocimiento gracias a la interacción entre los grupos funcionales y las comunidades de práctica.

La figura anterior describe el modelo 3, muestra a miembros de dos comunidades y muestra que la creación de conocimiento se lleva a cabo

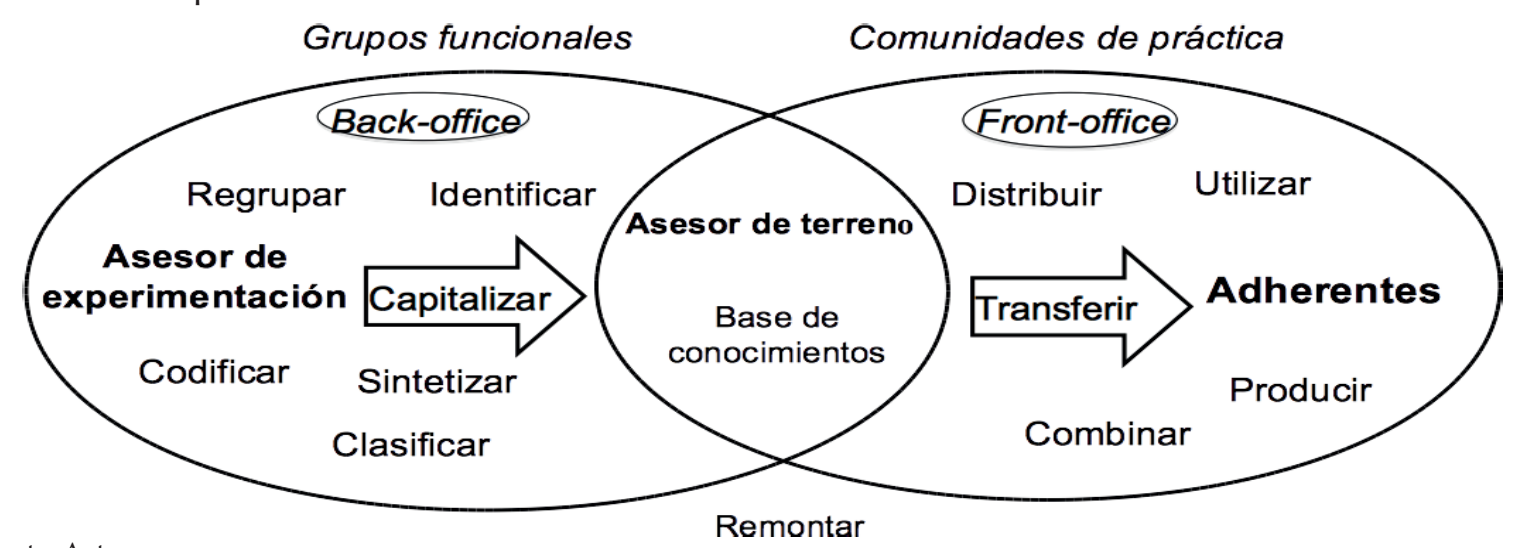

Fuente: Autor

Remontar

Figura 4. Creación y difusión de conocimiento en la actividad de asistencia técnica en las cooperativas agrarias en Francia 
en un proceso en espiral (Nonaka y Takeuchi 1995). Esto implica que el conocimiento tácito de los agricultores se codifica en los grupos funcionales, y que el conocimiento ya codificado por los grupos funcionales se utiliza para crear nuevo conocimiento tácito. De hecho, el nuevo conocimiento codificado (boletines, materiales de información, en particular) en los grupos funcionales se distribuyen a los miembros por medio de los asesores o responsables de portafolio.

Gracias a la intervención de los técnicos la intermediación de las dos comunidades se realiza de manera top-down y bottom-up. Este proceso es posible debido a la especificidad de la gestión híbrida de grupos cooperativos agrarios. De este modo, se demuestra que a través de la práctica de asistencia técnica, las cooperativas pueden integrar a los miembros en la toma de decisiones y en especial en la creación de la asistencia técnica, que está implicada en la creación de conocimiento dentro de los grupos cooperativos agrarios. Con el fin de evaluar el rol de la asistencia técnica en la resolución de la crisis de identidad de los grupos cooperativos agrarios, decidimos cuantificar dos indicadores de medición de las intereacciones en las dos comunidades cognitivas identificados anteriormente. Asi, medimos el nivel de intervención (frecuencia de interacciones) y el nivel de desarrollo de la estrategia de la asistencia técnica (formas de intercambio). A continuación presentamos la tabla 3 recapitulativa de los resultados. Tres tendencias se observan en esta tabla:

- Una buena posición de GC4 tanto en lo que se refiere a la propuesta de servicios de

Tabla 3. Resultados para los grupos cooperativos agrarios en Francia.

\begin{tabular}{lcc}
\hline Nivel & $\begin{array}{c}\text { Nivel de intervención } \\
\text { de los técnicos }\end{array}$ & $\begin{array}{c}\text { Nivel de desarrollo de la estrategia } \\
\text { de asistencia técnica }\end{array}$ \\
\hline Elevada & GC3 & GC4 \\
Relativamente & GC4 & GC1 \\
elevada & GC5 & GC5 \\
Intermedia & GC1 & GC3 \\
Moderada & GC2 & GC2 \\
\hline
\end{tabular}

asistencia técnica ofrecidos por el responsable de portafolio más que sobre las OAD y la estrategia de la organización de la asistencia técnica parece ser favorable para la interacción entre los grupos funcionales y comunidades cognitivas. Esta interacción también ayuda a mantener el vínculo cooperativa- asociado. - Las dificultades de GC2 en los dos indicadores. Esta situación no es propicia para la interacción entre los grupos funcionales y las comunidades cognitivas. De hecho, GC2 es un nuevo grupo que presenta problemas de gobernanza. Se observa la dificultad de ponerse de acuerdo sobre la estrategia organizacional de las dos cooperativas que se formaron GC2 después de una fusión. La comunicación entre las dos comunidades no es eficiente debido a que el proceso de traducción se hace difícil. - La posición intermedia de GC5, quien se situa en un nivel medio en relación con otros grupos cooperativos agrarios. En este contexto, la interacción entre los grupos funcionales y comunidades de práctica también permite la difusión y creación de conocimiento. Sin embargo, el grupo puede mejorar aún más la relación con los asociados para facilitar la negociación de las creencias y los intereses de ambas comunidades. Una vez identificado el nivel de cada grupo cooperativo en relación con estos dos indicadores, se reconstruye la imagen inspirada en la obra de Cohendet y Diani (2003) para representar la identidad cooperativa de cada uno de estos grupos (Tabla 4).

Tabla 4. Identidad cooperativa según la calidad de interacciones entre las comunidades cognitivas.

Nivel de interven- Nivel de intervención de ción de los téc- los técnicos (frecuencia nicos (frecuencia de interacciones) de interacciones) Elevado Débil

\begin{tabular}{|c|c|}
\hline $\begin{array}{l}\text { Nivel de desarrollo } \\
\text { de la estrategia de Identidad débil } \\
\text { asistencia tecnica GC2 } \\
\text { (formas de } \\
\text { intercambio) } \\
\text { Débil }\end{array}$ & $\begin{array}{l}\text { Identidad (tácita) fuerte } \\
\text { GC3 }\end{array}$ \\
\hline $\begin{array}{l}\text { Nivel de desarro- } \\
\text { llo de la estrategia Identidad (codifica- } \\
\text { de la asistencia da) fuerte } \\
\text { técnica (formas GC1 } \\
\text { de intercambio) } \\
\text { Elevado }\end{array}$ & $\begin{array}{l}\text { Identidad fuerte } \\
\text { GC4 }\end{array}$ \\
\hline
\end{tabular}


Esta tabla, inspirada en la literatura no permite posicionar nuestros 5 casos. En efecto, GC5, presenta características intermedias. Por tanto, proponemos una representación gráfica en forma de ejes en las que ubicamos nuestros 5 casos de estudio (nivel de intervención en abscisas y nivel de desarrollo de la estrategia en ordenadas).

Al final, los criterios de selección que nos hemos fijado para nuestra muestra (incluida la variedad) son relevantes en la medida en que nos encontramos en los resultados de todas las situaciones posibles de identidad cooperativa.

Esto también nos permitió identificar nuevos casos no especificados en la literatura (a través de la identidad de grupo).

- En la figura 5, se identifican tres grupos que se diferencian de los otros. GC4 que tiene ambos nivelesaltos, GC5 queestá enel medio del eje y GC2 que tiene los dos niveles bajos. - Si hacemos un análisis con respecto al tipo de producción (polivalente / especializado), encontramos diferencias. Por un lado, los grupos especializados (en rojo en la figura 5) están en un nivel limitado de desarrollo de la estrategia de consultoría (más bien baja). Sin embargo, en encuentran en las dos situaciones posibles de nivel de intervención; GC3 en el eje elevado y GC2 en el bajo. Por otro lado, los grupos polivalentes presentan más bien un nivel fuerte de desarrollo de la estrategia de la asistencia técnica y un nivel de intervención variable. De hecho, tres grupos polivalentes se encuentran en los tres grados del eje (mayor para GC4, medio para GC5 y débil para GC1.

\section{CONCLUSIONES}

Nuestra investigación ha contribuido a una mejor comprensión del rol de la asistencia técnica y sus efectos como mecanismo de gestión de las cooperativas agrarias. El impacto del desarrollo de esta actividad en los grupos cooperativos fue confirmado empíricamente, tanto en la dimensión contractual como en la dimensión cognitiva. De hecho, presentamos como consecuencia del aumento de tamaño de las cooperativas agrarias la ruptura del vínculo cooperativaasociado. Este contexto causa una crisis de identidad que planteaba cuestionamientos de su nueva gobernanza hibrida. Varios estudios han puesto en duda la idoneidad de la gestión de estas nuevas estructuras(gruposcooperativos)(Chiffoleau, 1999; Côté, 2005, 2009; Couret, 2002; Deroy y Thénot, 2012; Forestier y Mauget,

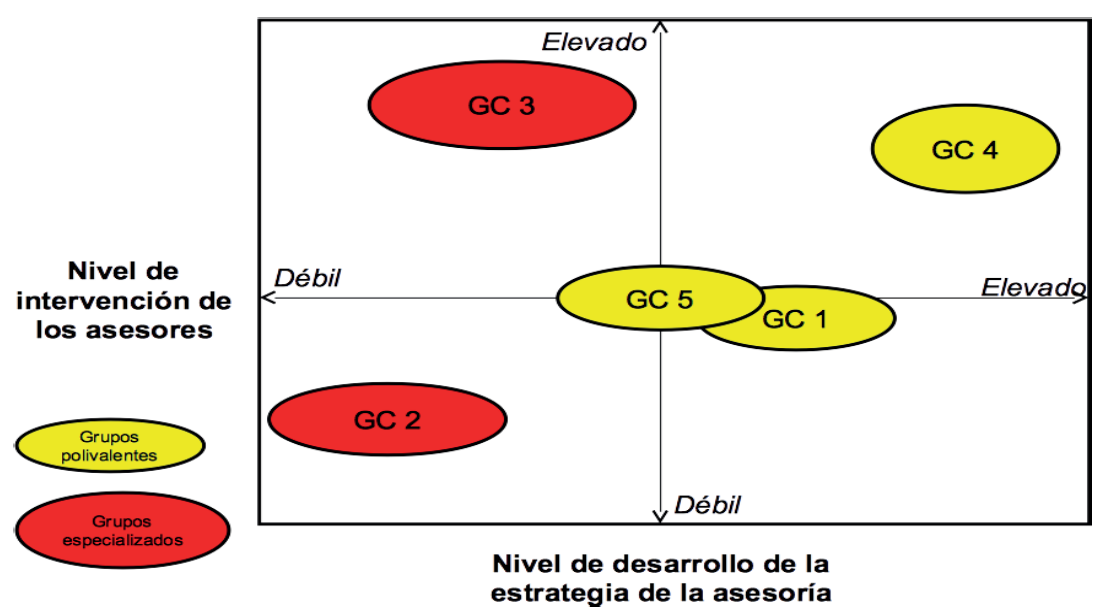

Figura 4. Identidad cooperativa des grupos cooperativos agrarios 
2000; Gianfaldoni y Richez-Battesti, 2008). Mientras que algunos hacen hincapié en la importancia de reforzar el vinculo (Côté, 2005), ninguno ha investigado el rol de la asistencia técnica en este nuevo contexto.

Nuestro enfoque es original porque puede revelar la solución de la crisis de identidad a través de la asistencia técnica. Asi, nuestros resultados confirman el rol de la asistencia técnica como mecanismo de gobernanza cooperativa, ya que contribuye a la creación de valor en la empresa a través de las prácticas asociativas.

Entre estas prácticas se distinguen: las prácticas de formación e información que facilitan los intercambios entre técnicos y miembros, dando lugar a la discusión sobre la estrategia de la organizacion y de la asesoría y la práctica de consulta que permite identificar las necesidades de los asociados. Sin embargo, los resultados de nuestros estudios de caso muestran que la información es la práctica asociativa más desarrollada, seguida por la formación y la consulta. Hemos demostrado que, si bien la práctica de la consulta debe ser lo más importante en este tipo de organizaciones, es la menos desarrollada.

\section{REFERENCIAS}

Barkaoui, A. and Butault, J. 2004. Impacts sur l'offre des régions françaises des différentes options de la réforme de la PAC de 2003. INRA Sciences Sociales 4-5/03, pp. 6.

Brown, J. and Duguid, P. 1991. "Organizational Learning and Communities of practice: Toward a Unified View of Working, Learning and Innovation", Organization Science. Vol. 2, $\mathrm{n}^{\circ} 1$, pp. 40-57. https://doi.org/10.1287/orsc.2.1.40
Charreaux, G. 2002. "Quelle théorie pour la gouvernance? De la gouvernance partenariale à la gouvernance cognitive », cahier de recherche, Fargo.

Chiffoleau, Y. 1999. Je ne vais pas aux AG et pourtant j'ai des idées: pour une coopération rurale en Languedoc-Roussillon. Revue des études coopératives mutualistes et associatives, 273, pp. 27-39.

Cohendet, P. and Diani, M. 2003. L'organisation comme une communauté de communautés: croyances collectives et culture d'entreprise. Revue d'Economie Politique, vol. 113, $\mathrm{n}^{\circ}$, pp. 697-721. https://doi. org/ $10.3917 /$ redp.135.0697

Cohendet, P., Créplet, F. and Dupouët, $O$. 2003. "Innovation organisationnelle, communautés de pratique et communautés épistémiques: le cas de Linux, Revue française de gestion, $\mathrm{n}^{\circ}$ 146, pp. 99-121.

Coté, D. 2005. Loyauté et identité coopérative - L'implantation d'un nouveau paradigme coopérative, Recma no 295, pp.50-69.

Côté, D. 2007. "Fondements d'un nouveau paradigme coopératif (NPC): quels incitatits pour les acteurs clés?" working paper, HEC Montréal, janvier.

Côté, D. 2009. "Le mode d'organisation coopérative au 21e siècle: un nouveau paradigme coopératif face à la crise identitaire", Revue Internationale de Projectique, $n^{\circ} 2$ - 2009/2, pp.61-84.

Couret, F. 2002. Principe démocratique et décision en coopérative, RECMA-Revue Internationale de I’Economie Sociale, n²85, pp.13-26.

Deroy, X. and Thenot, M. 2012. $L^{\prime}$ interaction des logiques coopérative et de marché : quelle évolution pour le modèle coopératif agricole français ? Le cas de Champagne Céréales. In Brassard, M.-J., Molina, E. (dir.) 2012 « L'étonnant pouvoir des coopératives" Textes choisis de l'appel international de propositions, Québec, Sommet international descoopératives, pp. 665. 
Duvaleix, S., Cordier, J. and Hovelaque, V. 2003. Vers un nouvel engagement coopératif dans le secteur laitier: l'économie sociale en mouvement, Revue internationale de l'économie sociale $\mathrm{N}^{\circ}$ 288, pp. 37-47.

Faure, G., Desjeux, Y. and Gasselin, P. 2011. Revue bibliographique sur les recherches menées dans le monde sur le conseil en agriculture », Cahier Agricole, vol. 20, pp.327-342.

Forestier, M. and Mauget, R. 2000. De la coopérative au groupe coopératif agro-alimentaire, 1ere partie : de la valeur pour I'usager à la valeur pour l'actionnaire? », Recma, $n^{\circ} 78$.

Forestier, M., Mauget, R. 2001. De la coopérative au groupe agroalimentaire», Recma, n²79, pp.60-70.

Gianfaldoni, P. and Richez-Battesti, N. 2008. La gouvernance partenariale des banques coopératives françaises, rapport de recherche financé par la DIIESES, Mars.

GNC, 2010. Top 100 des entreprises coopératives et panorama sectoriel, les entreprises coopératives acteurs économiques incontournables, étude réalisé par le Groupement National de la Coopération (GNC), pp. 51.

Lave, J. and Wenger, E. 1991. "Situated learning: legitimate peripheral participation', collection Learning in Doing: Social, Cognitive and Computational Perspectives", Cambridge, Cambridge University Press. https://doi. org/10.1017/CBO9780511815355

Le Theule, F. y Litvan, D. 1993. La réforme de la PAC: analyse d'une négociation communautaire », Revue française de science politique, 43e annẻe, $n^{\circ} 5$, 1993, pp. 755-787.

Lefer, H. 1997. Compétitivité et protection de l'environnement. L'apport des modèles bioéconomiques dans l'évaluation des politiques agricoles, Thèse de doctorat en sciences économiques, Université de Sciences et Technologies de Lille (USTL, Lille 1), janvier, pp. 181.
Meynard, J. 1998. L'emploi de plantes transgéniques va-t-il obliger les agriculteurs à modifier leurs pratiques?, Dossier publié par I'INRA, mai, pp. 5.

Nicolas, P. 1993. Règles et principes dans les sociétés coopératives agricoles françaises: évolution du droit et des pratiques de 1960 à 1992, in Zevi A.et MonzonCampos J.L. (dir.), Coopératives, marchés, principes coopératifs, De Boeck Université, pp.155-185.

Nonaka, I. and Takeuchi, H. 1995. "The knowledge-creating company". New York: Oxford

Rémy, J., Brives, H. and Lémery, B. 2006. "Conseiller en agriculture », Editions Quae, Versailles (FRA), pp. 276.

Vargas Prieto, A. 2013. Le rôle du conseil agricole dans la gouvernance coopérative ", Thèse de Doctorat en Sciences Economiques université MONTESQUIEU - BORDEAUX IV. Soutenue le 6 septembre, pp. 416.

Vargas Prieto, A. 2014. La crisis de identidad de las cooperativas agrarias en Francia. Cooperativismo \& Desarrollo, 22(105), 31-46. doi: http://dx.doi. org/10.16925/co.v22 i 105.1032

Vargas Prieto, A. 2015. Hacia un nuevo modelo de gestión del conocimiento caracterizado por la interacción de comunidades cognitivas. Universidad \& Empresa, 17(28), 219-234. https://doi.org/10.12804/ rev.univ.empresa.28.2015.10

Wenger, E. 1998. "Communities of practice: learning, meaning and identity", Cambridge, Cambridge university press. https://doi. org/10.1017/CBO9780511803932 